\title{
ESTABLISHMENT OF RADIO STANDARDS OF FRE- QUENCY BY THE USE OF A HARMONIC AMPLIFIER
}

\author{
By C. B. Jolliffe and Grace Hazen
}

\begin{abstract}
A method is described for measuring the ratio of a radio to an audio-frequency by the use of a harmonic amplifier. The harmonic amplifier makes it possible to use harmonics of a very high order from a known low-frequency source, such as a standard tuning fork. The method consists essentially of the production of harmonics of the fundamental frequency of an alternating current by means of the nonlinear characteristics of electron tubes, the selection of any desired harmonic by means of tuned circuits, and its amplification to sufficient power to operate a standard frequency meter (wave meter). Any harmonic of the source may be selected, and thus from a known audio-frequency source a frequency meter may be standardized throughout its entire range.

The harmonic amplifier consists of two units, one having a range from 8 to 450 $\mathrm{kc}$, the other from 400 to $4,000 \mathrm{kc}$. The first unit supplies a harmonic which is used as the fundamental for the second unit. The harmonic amplifier is given a preliminary calibration, so that the harmonic multiples can be readily determined.

A fixed frequency generator, such as a piezo oscillator, may be standardized with the aid of an auxiliary device to determine the frequency of the beat note occurring between a harmonic of the amplifier and the fixed frequency. The device used for this purpose is a sonometer. It consists of a steel piano wire mounted horizontally across two movable knife-edges with a known tension applied. The beat-note frequency is applied to the wire through a telephone receiver. The wire vibrates when its frequency is equal to the applied frequency. The frequency of vibration may be computed from the length, tension, and mass per unit length of the wire. Two audio-frequencies may be compared very accurately by the use of the harmonic amplifier and sonometer.
\end{abstract}

\section{CONTENTS}

II. Description of the method

1. Circuits and apparatus

2. Auxiliary apparatus for standardization of a fixed-frequency generator.

3. Fundamental frequency source

4. Preliminary calibration of a harmonic amplifier._._..... 184

III. Frequency meter (wave meter) standardization

1. Method of taking observations_._. 184

2. Frequency meter.

3. Results_._.

IV. Piezo oscillator standardization

1. Method_...

2. Results

V. Comparison of two audio-frequencies.

VI. Summary $94252^{\circ}-26$ 


\section{INTRODUCTION}

In the establishment of standards of radio-frequency several methods have been used by the Bureau of Standards. ${ }^{1}$ The harmonics from an electrically driven standard tuning fork give accurate frequency points and have been used by various laboratories. A very complete arrangement for obtaining and using harmonics from a tuning fork is maintained by the Bell Telephone Laboratories (Inc.), and has been described by them. ${ }^{2}$ The method here described is similar to and was suggested by that system. It is simpler, however, and has been adapted to the needs of this laboratory and has proved to be a very satisfactory method of frequency standardization.

The method consists, essentially, of the production of harmonics from a fundamental frequency source by means of the nonlinear characteristics of electron tubes, the selection of desired harmonics by means of tuned circuits, and the amplification of a desired harmonic to sufficient power to operate a standard frequency meter (wave meter). Any harmonic of the source may be selected, and thus from a known audio-frequency source a frequency meter may be standardized throughout its entire range. Any fixed frequency generator, such as a piezo oscillator, may be standardized with the aid of an auxiliary device to determine the frequency of the audio beat between a harmonic of the amplifier and the fixed frequency.

\section{DESCRIPTION OF METHOD}

The harmonic amplifier consists of two units, one for the production, selection, and amplification of the harmonios of frequencies from 8 to $450 \mathrm{kc}$ and the other for the higher harmonics covering a range from 400 to $4,000 \mathrm{kc}$. The first unit supplies a harmonic which is used as the fundamental for the second unit. The frequency meter is coupled directly to the last stage of the amplifier, and the amplification is such that sufficient power is obtained so that the resonance indicator of the frequency meter may be used directly; that is, as it is normally used in frequency measuring work. The harmonic amplifier may be used with any low-frequency source as a basic standard. A 1,004-cycle standard tuning fork was used as the fundamental frequency source in the greater part of this frequency standardization. A 100-cycle fork was used to give a larger number of points below

1 A general summary of the various methods is given in the article "Reducing the guesswork in tuning," J. H. Dellinger, Radio Broadcast, 3, p. 241; July, 1923. See also "A method of measuring very short radio wave lengths and their use in frequency standardization," F. W. Dunmore and F. H. Engel, Proc. Inst. of Radio Eng., 11, p. 467; October, 1923; “Theory of Determination of Ultra-Radio Frequencies by Standing Waves on Wires," A. Hund; B. S. Sci. Paper No. 491, 1924; "Primary Radio-Frequency Standardization by Use of the Cathode-Ray Oscillograph," Grace Hazen and Frieda Kenyon, B. S. Sci. Paper No. 489, 1924.

${ }^{2}$ Horton, Ricker, and Marrison, Bell System Tech. Jour., June, 1923, p. 730, Kendall. U. S. Patent No. 1446752. 
$60 \mathrm{kc}$. A sonometer was used as an auxiliary in standardizing piezo oscillators to determine the difference in frequency between the nearest harmonic of the fundamental frequency as given in the harmonic amplifier and the unknown fixed frequency.

\section{CIRCUITS AND APPARATUS}

The first unit of the harmonic amplifier (fig. 1) used for the lowfrequency standardization is made up of four stages. The output from the circuit controlled by the tuning fork or fundamental frequency source is applied to tube 1 through an audio-frequency (ironcored) transformer. This tube is coupled to tube 2 by an intermediate-frequency transformer (iron-cored). Tubes 2 and 3 are inductively coupled through tuned plate and grid circuits, respectively. Tubes 3 and 4 are resistance coupled through 100,000-ohm resistors.

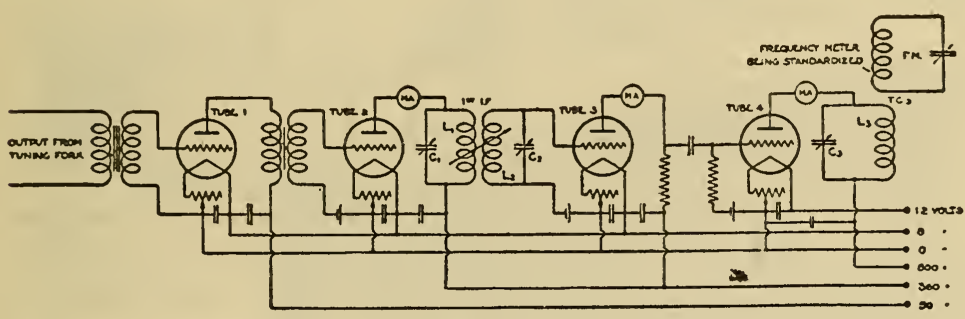

FIG. 1.-Circuit diagram of harmonic amplifier as used to obtain low frequencies $(8-450 k c)$

The tubes used are 5-watt power tubes with the exception of tube 4 , which is a 50-watt tube. Where grid batteries are indicated the voltage is chosen of such an amount that the direct plate current is nearly zero with no impressed a. c. voltage; that is, the distortion is produced by the lower curvature of the grid voltage-plate current characteristic.

The values of capacity and inductance of the first tuned stage referred to as the first intermediate frequency (1st $I$. $F$., fig. 1) were chosen to cover the range of frequencies 8 to $15 \mathrm{kc}$, which gives a sufficient number of points for the frequency-meter standardization. Coils having an inductance of approximately 40 millihenries (outside diameter of winding, 6 inches; width of winding, 1 inch) are used for coupling coils $L_{1}$ and $L_{2}$. Condensers $C_{1}$ and $C_{2}$ are 0.005 $\mu \mathrm{f}$ variable air condensers with a switch, making possible the addition of a fixed condenser for additional capacity. The tuned circuit in series with the plate of tube 4 is arranged to cover the frequency range 8 to $450 \mathrm{kc}$. The inductance coils used varied from 0.01 to $24 \mathrm{mh}$, and have large diameters approximately the size of the coils 
of the frequency meter to be coupled to them. The capacity was made up of a parallel combination of fixed and variable condensers and was continuously variable up to $0.01 \mu f$. For the range 8 to 450 $\mathrm{kc}$, the frequency meter being standardized is coupled directly to coil $L_{3}$, the coupling being adjusted to give a sufficient indication on the indicator.

The second unit, which is a 2-tube resistance-coupled amplifier, is used to extend the range to $4,000 \mathrm{kc}$. (Fig. 2.) This unit is identical with the last two stages of the low-frequency unit with the exception of the inductances and capacities of the circuits. When this unit is used the coil $L_{3}$ is replaced by a coil $L_{3}^{\prime}$ of small diameter similar to coils $L_{1}$ and $L_{2}$, but having an inductance of approximately $22 \mathrm{mh}$. This coil has the advantage orer the larger size coil of producing a more concentrated field and reduces the interaction with $L_{1}$. This unit is coupled to the first unit (fig. 1),

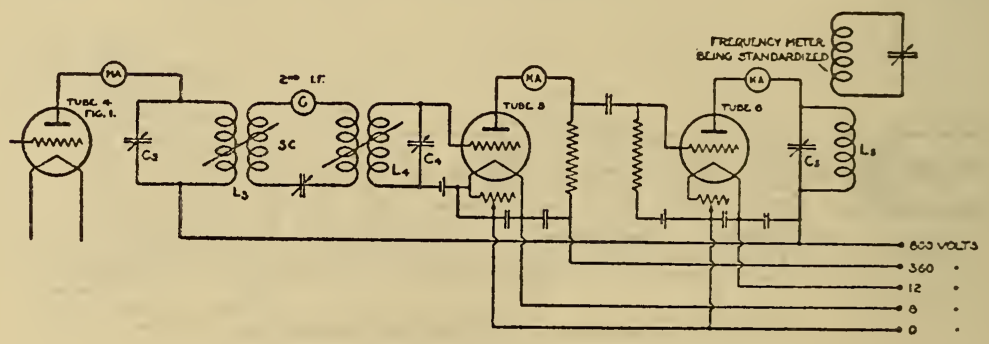

FIG. 2.-Extension of harmonic amplifier shown in Figure 1 to obtain frequencies to $4,000 \mathrm{kc}$

through a tuned selector circuit, $S C$, by means of inductance $L_{4}$ which is identical with $L_{3}^{\prime}$. The capacity, $C_{4}$, in the grid circuit of tube 5 consists of fixed and variable condensers continuously rariable up to $0.0045 \mu \mathrm{f}$. This gives a range from approximately 18 to $100 \mathrm{kc}$ for the second intermediate frequency ( $2 n d I . F$.) values.

The tuned selector circuit ${ }^{3}$ through which the two units are coupled consists of a condenser, coil, and resonance indicating derice (thermogalvanometer). This circuit serves to suppress other harmonics than the one selected and when calibrated makes it possible to set the circuit (2nd I. F.) to any desired harmonic of the first intermediate frequency, thus making the identification of the harmonic in the final stage fairly simple.

A $0.00025 \mu \mathrm{f}$ condenser, $C_{5}$, and coils, $L_{5}$, of proper inductance and diameter to be in resonance and give good coupling with the frequency meter under standardization, are used in the plate circuit of the last tube. All battery leads are by-passed with large condensers (about $1 \mu \mathrm{f}$ ).

A Bureau of Standards type L wave meter was used for this purpose. 
Scientific Papers of the Bureau of Standards, Vol. 21

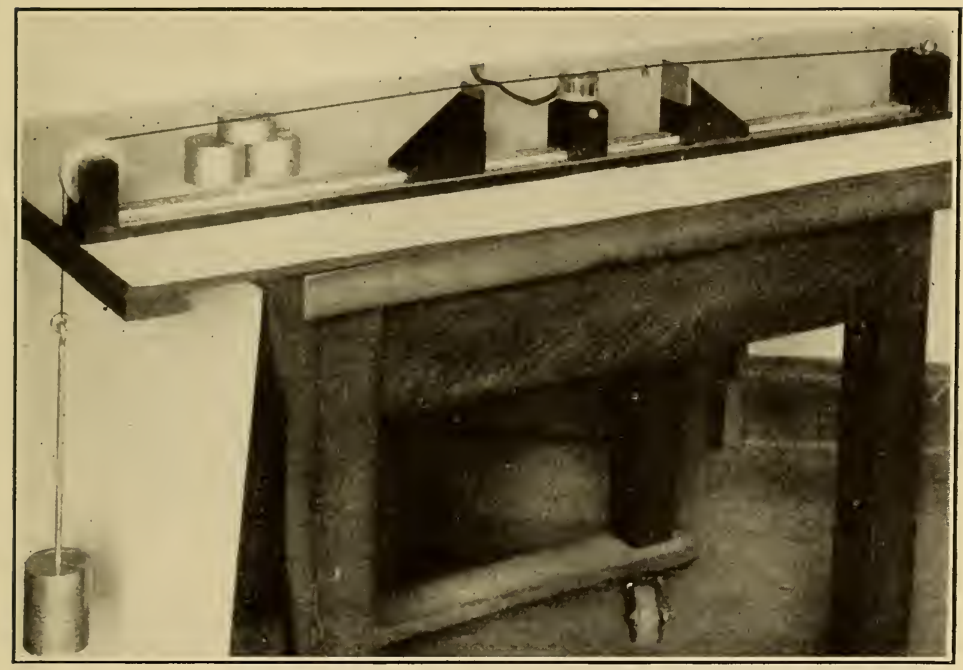

FIG. 3.-Sonometer used for the determination of the frequency of the beat note produced by two radio frequencies 
Scientific Papers of the Bureau of Standards, Vol. 21

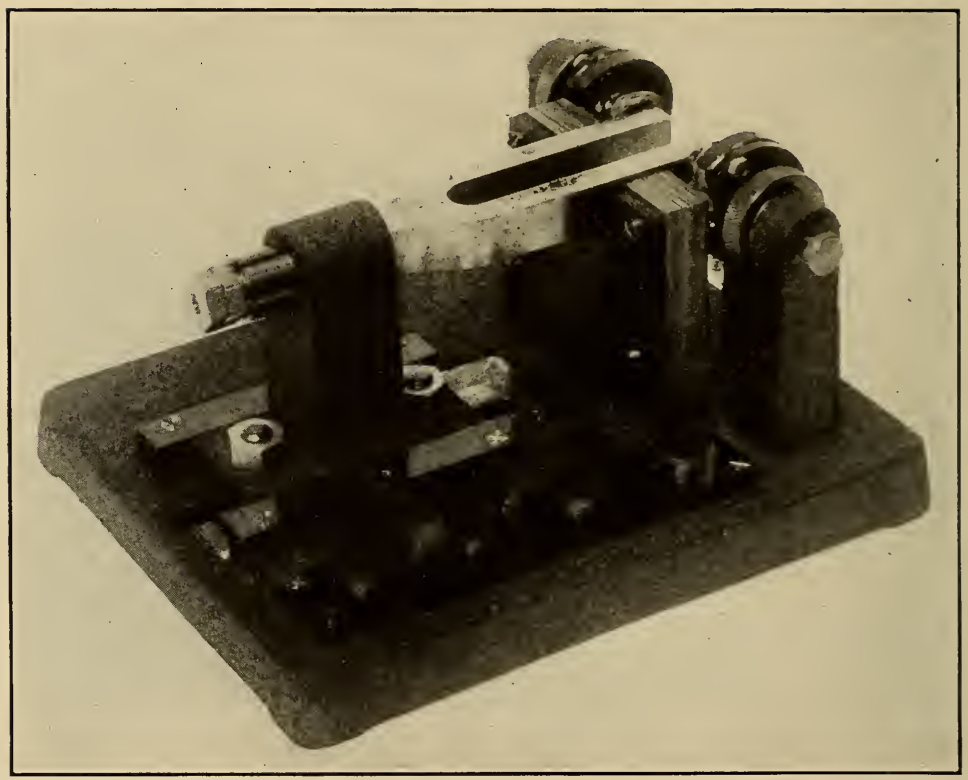

FIG. 4.-Mounted tuning fork used as the fundamental frequency source 


\section{AUXILIARY APPARATUS FOR STANDARDIZATION OF A FIXED- FREQUENCY GENERATOR}

An auxiliary device used with the harmonic amplifier to measure a fixed-frequency generator is a sonometer (fig. 3) or monochord having a range from $\$ 0$ to over 3,000 cycles. It consists of a steel piano wire (approximately $0.026 \mathrm{~cm}$ diameter) mounted in a horizontal position and stretched over two brass knife-edges with a known weight giving a definite tension to the wire. The frequency at which the wire vibrates is computed from $f=\frac{1}{2 L} \sqrt{\frac{T}{M}} . L=$ length between knife-edges; $T=$ tension; $M=$ mass of wire per unit length. To avoid loss in tension due to friction in applying weights, the wire is passed over a ball-bearing pulley. The calibration of the sonometer was checked at several points against a tuning fork of known frequency.

The beat-note frequency to be measured is applied to the sonometer through a telephone receiver magnet mounted at a small distance from the wire. As the length between the knife-edges is varied and the natural period of vibration of the wire approaches the frequency in the telephone, the wire will be set in motion due to the periodic attraction of the steel wire by the telephone magnet.

\section{FUNDAMENTAL FREQUENCY SOURCE}

The 1,000-cycle ${ }^{4}$ standard tuning forks used in this work (fig. 4) were standardized by the sound section of this bureau by comparison with a standard of time. The frequency values for the 100-cycle fork were obtained by comparison with a standard quarter-meter gravity pendulum loaned to the bureau by the Coast and Geodetic Survey. ${ }^{5}$

The electron-tube drive of the 1,000-cycle tuning forks is one in which the reactance between plate and grid is wholly mechanical as in the drive described by Eccles and Jordan. ${ }^{6}$ This avoids any possibility of the circuit producing oscillations independent of those actuated by the tuning fork. The 1,004-cycle tuning fork used in this work was calibrated to \pm 0.12 of a cycle, or 0.01 per cent. It was corrected for temperature changes, using the correction factor of -0.011 per cent per ${ }^{\circ} \mathrm{C}$. This correction factor is known to a certainty of \pm 0.004 per cent per ${ }^{\circ} \mathrm{C}$. The fork was kept at a nearly uniform temperature, so that this correction was small.

This circuit gives sufficient output for satisfactory operation of the harmonic amplifier as shown in Figure 1. For fundamental frequency sources of much less intensity than this further amplification

4 Two forks, one of 1,004 and one of 1,025 , were used in this work-the drives were identical.

${ }^{5}$ Acknowledgment is made to Dr. C. Moon for the use of this tuning fork and for his determinations of its frequency during its use.

- Eccles and Jordan, Electrician, June 20, 1919. 
was used in advance of tube 1 . With a 2-tube transformer-coupled amplifier an input of less than 0.4 of a volt was used satisfactorily on the harmonic amplifier.

\section{PRELIMINARY CALIBRATION OF THE AMPLIFIER}

The first step in the use of the harmonic amplifier is the approximate calibration of the intermediate frequency stages and the selector circuit. This can be done very easily by direct comparison with a calibrated frequency meter. However, if none is available the calibration of the circuit ( 1 st $I$. $F$.) may be obtained indirectly. Since no great accuracy is needed to distinguish between harmonics of this frequency-that is, 8 or $9 \mathrm{kc}$-the frequency of the lowest harmonic possible from the inductance and capacity of the circuit can be computed from $f=\frac{1}{2 \pi \sqrt{L C}}$. The exact condenser setting for the harmonics so determined can be found by adjusting the condensers $C_{1}$ and $C_{2}$ to a maximum current in the plate circuit of tube 4 . Haring determined the lowest harmonic the higher harmonics can be found by slowly reducing the capacity until another change of deflection occurs in the milliammeters in the plate circuits of tubes 3 and 4. The condenser settings corresponding to these deflections are recorded for the successive harmonics of the fundamental.

The tuned circuit, $T C_{3}$, was calibrated by comparison with a previously aalibrated frequency meter. It may be done with the aid of the first intermediate frequency using the selector circuit or any resonance indicator to determine when $C_{3}$ is set to the same frequency as the first intermediate frequency. Computations may be made to obtain a rough check as in the previous calibration. The selector circuit may be calibrated in the same manner as any frequency meter.

\section{FREQUENCY METER STANDARDIZATION}

\section{METHOD OF TAKING OBSERVATIONS}

The procedure followed in the calibration of the frequency meter for the low frequencies with the harmonio amplifier is as follows: The circuit shown in Figure 1 is used. The first intermediate frequency stage is set to the lowest frequency desired on the frequency meter by use of the previous calibration of the harmonic amplifier and a coil $L_{3}$ of the proper inductance to tune to this frequency is used. The coupling between $L_{1}$ and $L_{2}$ is adjusted to gire a suitable plate current in tube 4 . The capacity of the condenser, $C_{3}$, and the frequency meter under standardization, coupled to $L_{3}$, are raried until a deflection in the frequency-meter indicator shows that the two circuits are near resonance. The frequency meter is tuned to 
resonance and $C_{3}$ adjusted to maximum deflection. Slight adjustments may be made in the coupling between $L_{1}$ and $L_{2}$ to increase the amount of amplification. However, care must be taken that this coupling is kept loose or the tuned circuits will not be effective as harmonic selectors. The frequency meter is then again tuned to resonance with the circuit $T C_{3}$. This should correspond to the second harmonic of the intermediate-frequency stage. It can be determined by referring to the preliminary calibration of the circuit $T C_{3}$.

After the setting of the frequency meter for the first harmonic is determined the procedure followed is simply to tune the frequencymeter condenser to the next higher harmonic, which is indicated by a very small deflection of the frequency-meter resonance indicator. Then $C_{3}$ is adjusted to the maximum deflection and the frequency meter is tuned to resonance with the circuit $T C_{3}$.

For example, if the first intermediate stage is set at the tenth harmonic with a 1,000-cycle fundamental, frequency values can be determined at 10,20,30, etc., kc. To obtain another series, the intermediate-frequency stage is set to $11 \mathrm{kc}$. and thus multiples of $11 \mathrm{kc}$. are found. This can be repeated using different intermediate frequencies until sufficient points are determined for a calibration curve.

For the higher frequency standardization the procedure is very similar to that for the lower frequency. The circuit shown in Figure 2 is added to that shown in Figure 1. The intermediate-frequency stage is set to a convenient harmonic; for example, $10 \mathrm{kc}$, and $C_{3}$ using $L_{3}^{\prime}$ is adjusted to any harmonic of it from the preliminary calibration. If the third is chosen, then the selector circuit, $S C$, is set at $30 \mathrm{kc}$ and $C_{3}$ is adjusted to give maximum deflection in the selector circuit indicator. The coil $L_{4}$ is next coupled ${ }^{7}$ to the selector circuit and $C_{4}$ is adjusted until in resonance with the selector circuit, as shown by a small dip in indicator of the selector circuit. The frequency meter is then coupled to $L_{5}$ and the same procedure followed as with the low-frequency standardization. It is well to overlap the lower-frequency calibration then the series can be followed straight through. Otherwise, it is necessary to make a preliminary curve or compute the frequency to check the first multiple of each harmonic series.

\section{FREQUENCY METER}

The harmonic amplifier has been used to standardize the standard frequency meters of the Bureau of Standards. ${ }^{8}$ The first

7 This coupling is critical and must be kept loose or the harmonic selected may be suppressed and side harmonics appear which make the determination of the final value difficult.

8 "The standard'wave meters of the Bureau of Standards, E. L. Hall." Sibley J. of Eng. 88, May, 1924. 
frequency meter standardized, $Z$, had a range from 12 to $3,100 \mathrm{kc}$. The frequency meter circuit contained a variable air condenser ${ }^{2}$ of $0.0012 \mu \mathrm{f}$, with four mica condensers of $0.001,0.002,0.004$, and 0.008 $\mu \mathrm{f}$ which may be connected in any parallel combination with the variable air condenser, and six interchangeable coils ${ }^{10}$ of 0.01 to 128 $\mathrm{mh}$ inductance. A sensitive millivoltmeter and crystal detector in series with a pick-up coil coupled to the coil of the frequency meter was used to indicate resonance. The frequency meter is always grounded during use.

Overlaps were obtained by using the 0.001 or 0.002 fixed condensers in parallel with the variable air condenser.

\section{RESULTS}

The number of frequency values which it is possible to determine using the harmonic amplifier is indicated by the table.

Summary of frequency values obtained in standardization of frequency meter $Z$ by the harmonic amplifier

\begin{tabular}{|c|c|c|c|c|c|}
\hline \multirow{2}{*}{ Coil number } & \multirow{2}{*}{$\begin{array}{l}\text { Induc- } \\
\text { tance }\end{array}$} & \multirow{2}{*}{$\begin{array}{l}\text { Frequency } \\
\text { range }\end{array}$} & \multicolumn{2}{|c|}{$\begin{array}{l}\text { Number of } \\
\text { observations }\end{array}$} & \multirow{2}{*}{$\begin{array}{c}\text { Differ- } \\
\text { ence in } \\
\text { frequency } \\
\text { between } \\
\text { obserra- } \\
\text { tions }\end{array}$} \\
\hline & & & $\begin{array}{l}1,000 \text { tun } \\
\text { ing fork }\end{array}$ & $\begin{array}{l}100 \text { tun- } \\
\text { ing fork }\end{array}$ & \\
\hline $1+0.001 \mu \mathrm{f}-$ & $\begin{array}{l}m h \\
0.01\end{array}$ & $\left\{\begin{array}{c}k c \\
1,600-3,080 \\
1,170-1,600\end{array}\right.$ & $\begin{array}{l}42 \\
31\end{array}$ & & $\begin{array}{r}\text { Per cent } \\
2.3 \\
3.3\end{array}$ \\
\hline $\begin{array}{l}2 \\
2+0.001 \mu \mathrm{f}- \\
2+0.002 \mu \mathrm{f}\end{array}$ & .05 & $\begin{array}{l}605-1,170 \\
444-610 \\
367-\quad 446\end{array}$ & $\begin{array}{l}45 \\
40 \\
31\end{array}$ & & $\begin{array}{r}2.2 \\
2.5 \\
3.2\end{array}$ \\
\hline $\begin{array}{l}3-0.001 \mu \mathrm{f}- \\
3+0.002 \mu \mathrm{f}-\cdots\end{array}$ & .36 & $\begin{array}{ll}231- & 440 \\
170- & 234 \\
141- & 171\end{array}$ & $\begin{array}{l}52 \\
37 \\
21\end{array}$ & - & $\begin{array}{l}1.9 \\
2.7 \\
4.7\end{array}$ \\
\hline $\begin{array}{l}4 \\
4+0.001 \mu f \\
4+0.002 \mu f_{-}\end{array}$ & 2.44 & $\begin{array}{lr}92- & 174 \\
68- & 93 \\
56- & 68\end{array}$ & $\begin{array}{l}43 \\
37 \\
21\end{array}$ & (n) & $\begin{array}{l}2.3 \\
2.7 \\
4.8\end{array}$ \\
\hline $\begin{array}{l}5 \\
5+0.001-0 \mathrm{f} \\
5+0.002 \mu \mathrm{f}\end{array}$ & 23 & $\begin{array}{ll}31- & 56 \\
23- & 31 \\
19- & 23\end{array}$ & $\begin{array}{r}30 \\
13 \\
6\end{array}$ & $\begin{array}{l}11 \\
21 \\
18\end{array}$ & $\begin{array}{l}2.4 \\
2.9 \\
4.2\end{array}$ \\
\hline 6........... & 128.0 & $12-\quad 23$ & 14 & 17 & 3.7 \\
\hline Total_. & & $12-3,080$ & 463 & 67 & 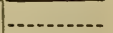 \\
\hline
\end{tabular}

Observations averaged erery $3^{\circ}$ with no fixed condenser, $5^{\circ}$ with $0.001 \mu \mathrm{f}$, and $7^{\circ}$ with $0.002 \mu \mathrm{f}$. It should be noted that at the higherfrequency ranges a much larger number of ralues can be obtained than are needed for the drawing of a calibration curve, since the harmonic from a 1,000-cycle fork, using all of the intermediate frequency points possible, will appear at rery small interrals on

- B. S. Cir. No. 74, Radio Instruments and Measurements.

${ }^{10}$,B. S. L. C. No. 103, Description of a series of single-layer inductance coils suitable for radio-frequency measurements. 
the frequency-meter condenser scale, especially when no fixed condensers are used. The same is true of the 100-cycle fork for the lower frequencies. It should be mentioned that for a frequency meter of the range here standardized it would be more convenient to use a 400-cycle tuning fork as an auxiliary fundamental frequency source instead of the 100-cycle standard, but none was available at this time.

The sources of error in standardization with the harmonic amplifier method are entirely those inherent in the fundamental frequency source and in the reading of the frequency meter under standardization. Since the frequency of the tuning fork may be determined more accurately than the present frequency meter can record, the precision of the frequency measurements can be increased as the design of frequency meters used are improved.

\section{PIEZO OSCILLATOR STANDARDIZATION}

The fixed-frequency generators standardized by the harmonic amplifier and sonometer were piezo oscillators. The piezo oscillator ${ }^{11}$ is a small, low-power generator with three frequencies determined and controlled by three modes of vibration of the quartz plate. Any one of these frequencies may be chosen by tuning the circuit to very approximately the frequency desired. Since the frequency values from the harmonic amplifier are fixed frequencies determined by the fundamental source it is obvious some variable device is necessary to standardize it against another fixed frequency. The sonometer described in Section II serves this purpose satisfactorily.

\section{METHOD}

In standardizing a piezo oscillator of unknown frequency an approximate value of its frequency is first determined by direct measurement with a standard-frequency meter. The harmonic amplifier is adjusted to the harmonic most nearly this frequency. A standard frequency meter is coupled to the output of the harmonic amplifier and tuned to resonance with it. The piezo oscillator is placed near the opposite (or grounded) end of the coil of the frequency meter which acts as a filter to other harmonics. The beat note between the harmonic selected and the fundamental of the puzo oscillator is amplified by a 2-tube resistance-coupled amplifier and the output measured by the sonometer in the following manner: The sonometer wire is stretched and its pitch roughly adjusted by ear to that of the beat note by changing the tension and length of the wire, then the telephone-receiver magnet in the output of the amplifier is

11 Uses and possibilities of piezo-electric oscillators. A. Hund. Proceedings of the Institute of Radio Engineers, 1926. Bureau of Standards Letter Circulars 183, 186, 187. 
placed under the wire, and the distance between the knife-edges is varied very slowly until the wire begins to vibrate. For weak signals a small paper rider is used to show the vibration of the wire, while for strong-beat notes a sharper maximum setting is obtained by setting on the maximum sound produced by the vibration of the wire. The length of the wire is measured and the frequency computed. This frequency gives the difference between the harmonic of the fundamental source and the frequency of the piezo oscillator. It is either a plus or minus correction, as is shown by the difference of the frequency of the piezo oscillator determined by the frequency meter reading, from the frequency of the harmonic given by the harmonic amplifier. If the harmonic and frequency meter readings are extremely close it is necessary to choose another harmonic setting to determine the direction of this correction; that is, whether the piezo oscillator frequency is above or below the harmonic frequency.

\section{RESULTS}

This method of standardizing a fixed-frequency oscillator was found very satisfactory by checking several piezo oscillators. The accuracy of the sonometer need not be high in order to obtain a high degree of precision in the radio range, for example, working with a beat note of 1,000 cycles the sonometer may have an error of 0.5 per cent. At any frequency over $100 \mathrm{kc}$ this would be less than 0.005 per cent error in the radio-frequency determination. Errors in the sonometer may be balanced out by using harmonics above and below the piezo oscillator frequency.

\section{COMPARISON OF TWO AUDIO-FREQUENCIES}

The harmonic amplifier and sonometer were also used to compare two audio-frequencies, 100 and 1,025 cycles. A beat note was obtained between a harmonic from the output of a 1,025-cycle electron tube driven tuning fork and the fundamental of a radiofrequency generator. This beat note was reduced to zero, this point being indicated by means of a sensitive milliammeter in the plate circuit of a detector tube. The output from the 100-cycle tuning fork was carried through the harmonic amplifier and a harmonic selected near the value of the one selected by the generator. The two frequencies were then combined in a tuned circuit and the beat note produced detected, amplified, and measured by means of the sonometer, as was done with the piezo oscillator. Several combinations of harmonics were used to obtain a mean value with a maximum error of less than 0.02 per cent. 


\section{SUMMARY}

This paper describes a harmonic amplifier and its application in establishing radio standards of frequency from an audio-frequency source. The results of one frequency meter standardization are summarized briefly. A sonometer, an auxiliary pitch-measuring derice, is described, and the method of using the harmonic amplifier with this auxiliary for the standardization of piezo oscillators and comparison of audio-frequencies is given.

The work has shown that radio-frequency meters may be standardized with high precision and ease from a fundamental audiofrequency by the use of a harmonic amplifier. The accuracy of the standardization is limited only by the accuracy of the fundamental frequency source and the precision and accuracy of the frequency meter. The harmonic amplifier is simple and rapid in operation. Fixed-frequency generators, such as piezo oscillators and electron tube driven tuning forks, may also be accurately and rapidly standardized by the use of the harmonic amplifier and the auxiliary sonometer.

Washington, March 23, 1926. 



